\title{
Letters
}

\section{A molecular biological approach to neuropathy of the urinary bladder/prostate in patients with spinal cord injury}

Spinal cord injury often results in neuropathic dysfunction of pelvic organs. Denervation/decentralization of the pelvic organs produces certain changes at the molecular level, which may be of clinical significance. For example, under experimental conditions, nerve growth factor expression in the ventral prostate is minimal under normal circumstances, whereas it is activated under unilateral denervation of the prostate in male Sprague-Dawley rats. The expression of nerve growth factor is upregulated in dorsal prostate in response to unilateral denervation. ${ }^{1}$ In clinical scenario, a retrospective analysis of the records from the Department of Veterans Affairs medical database for a seven-year period, 1986-1992, showed that severe paralysis due to myelopathy was a low risk factor for carcinoma of the prostate gland. ${ }^{2}$ If the molecular mechanisms(s) responsible for changes in urogenital organs after denervation can be identified, this might then pave the way for possible future development of new therapeutic strategies in able-bodied individuals.

Neuropathic bladder per se results in functional obstruction of the bladder outlet, e.g. detrusor-sphincter dyssynergia. Partial outlet obstruction of the rabbit urinary bladder causes significant changes in the status and expression of the mitochondrial genetic system. The expression of the mitochondiral and mitochondrial-related nuclear genes is no longer regulated coordinately in obstructed bladder. $^{3}$ Using cDNA probes to rabbit smooth muscle myosin heavy chain (MHC), Lin and McConnell ${ }^{4}$ studied the transcription of both total myosin heavy chain gene expression as well as the individual isoforms by Northern Blot and $\mathrm{S} 1$ nuclear protection assays respectively. Northern Blot analysis of the normal rabbit bladder demonstrated a high constitutive level of MHC expression. Shortly after the onset of obstruction, MHC expression was significantly down-regulated. By the third week, MHC expression tapered down to barely detectable levels. Nuclease protection assays, capable of simultaneously quantitating the individual isoforms, demonstrated a significant shift towards SM1 expression. Interestingly, the predominant SM1 expression pattern is also seen in the developing fetal rabbit bladder and in dedifferentiated smooth muscle cells in culture. It seems likely that obstruction in the bladder induces dedifferentiation of the smooth muscle cell which alters contractility as well as extracellular matrix expression, leading to altered bladder performance and decreased compliance.

Neuropathy of urinary bladder following spinal cord injury is reflected at the molecular level by certain changes which alter the structure and function of the urinary bladder. For example, gamma-smooth muscle isoactin gene is expressed 4.3 times that of alpha-smooth muscle isoactin in control rat bladder smooth muscle. Four weeks after T10 spinal cord injury, gamma:alpha -isocatin gene expression changes to a 10:1 ratio. ${ }^{5}$

If we can (1) identify the changes taking place at the molecular level in the neuropathic bladder of patients disabled due to spinal cord injury, and (2) develop innovative molecular biological strategies to (i) block the unwanted changes taking place in the neuropathic bladder, or (ii) mimic what is happening in the normally innervated urinary bladder, then such novel techniques may prove to be of therapeutic value to the patients disabled due to spinal cord injury. In experimental studies, application of antisense Transforming Growth Factor Beta ${ }_{1}$ (TGF-Beta $)_{1}$ oligodeoxynucleotides on injured urethrae in rats, resulted in (1) inhibition of expression of TGF-Beta 1 mRNA, and (2) consequently, prevention of urethral stricture formation. ${ }^{6}$

Antisense oligonucleotide therapy represents a new way of blocking protein function by preventing the translation of messenger RNA (mRNA) into protein. If the nucleic acid sequence encoding a protein is known, molecules can be designed to bind the corresponding mRNA, thereby inhibiting the production of the protein. The nucleotide sequence of DNA or RNA that contains the information for the amino acid sequence of the protein is called the sense strand. In double-stranded DNA, the other nucleotide sequence is complementary to the sense strand and is called the anti-sense strand. It is possible to synthesise antisense strand oligonucleotides that bind the sense strand of RNA or DNA with a high degree of specificity. The antisense strand oligonucleotides bind to the mRNA and block the synthesis of the encoded protein product at the ribosomes.

Problems with antisense-oligonucleotide therapy include the destruction of the oligonucleotides by DNase, which is ubiquitous in the body; the large doses required for a therapeutic response; the difficulty in directing oligonucleotides to particular cells; and the need for parenteral administration. Another major problem with antisense oligonucleotides, which should be mentioned, is the immune response against oligonucleotides. But the oligonucleotide therapy does not have the safety and efficacy issues associated with expressed-vector gene therapy. ${ }^{7}$ One of the limitations to the clinical application of antisense technology viz. difficulties in the targeting of the antisense oligodeoxynucleotide to a specific organ may be overcome in case of the urinary bladder, as the bladder is readily accessible for direct intravesical instillation via a urethral catheter. Such an innovative technology of clinical application of antisense-oligonucleotide therapy has potential economic benefits not only to the patients with spinal cord injury, but also to the organisations developing this unconventional, and pioneering therapeutic modality, as the commercial value of a clinically successful endeavour may be enormous.

S Vaidyanathan MBBS MCh PhD, Registrar KR Krishnan MB FRCS, Honorary Consultant Regional Spinal Injuries Centre District General Hospital Town Lane Southport Merseyside PR8 6PN United Kingdom 


\section{References}

1 Guan X, McKenna KE, McVary KT. Expression of nerve growth factor in pelvic organs of male rat in response to denervation. Journal of Urology, volume 155, May 1996; Supplement, Abstract number 900 .

2 Frisbie JH, Binard J. Low prevalence of prostatic cancer among myelopathy patients. Journal of American Paraplegia Society, volume 17, July 1994, $148-149$.

3 Nevel-McGarvey CA, Levin RM, Hudson AP. Transcription of mitochondrial and mitochondrial-related nuclear genes in rabbit bladder tissue after partial outlet obstruction. Journal of Urology, volume 155, May 1996; Supplement, Abstract number 102 .

4 Lin VK, McConnell JD. Molecular aspects of bladder outlet obstruction. In: Muscle, Matrix, and Bladder Function, Edited by S. Zderic, Plenum Press, New York, 1995; pp 65-79.

5 Rivas DA et al. Bladder smooth muscle isoactin gene expression in the rat model of spinal cord injury. Journal of Urology 1996; 155: $597 \mathrm{~A}$.

6 Seo I-Y, Kim J-S, Chung H-T, Rim J-S. Application of antisense TGF-B ${ }_{1}$ oligonucleotides on injured urethrae prevents urethral stricture in rats. Journal of Urology 1996; 155: 504A.

7 Askari FK, McDonnell WM. Antisense-oligonucleotide therapy. The New England Journal of Medicine 1996; 334: 316-318. 